\title{
The Effects of Aortic Clamping Strategy on Myocardial Protection and Early Postoperative Outcomes During Coronary Artery Bypass Grafting Operations
}

\author{
Cuneyt Eris, MD, ${ }^{1}$ Burak Erdolu, MD,${ }^{1}$ Mesut Engin, MD,${ }^{1}$ Ahmet Kagan As, MD,${ }^{1}$ Yasemin Ustundag, $\mathrm{MD}^{2}$ \\ ${ }^{1}$ University of Health Sciences, Bursa Yuksek Ihtisas Training and Research Hospital, Department of Cardiovascular Surgery, Mimar \\ Sinan Town. Yıldırım/BURSA, Turkey; ${ }^{2}$ University of Health Sciences, Bursa Yuksek Ihtisas Training and Research Hospital, \\ Department of Clinical Biochemistry, Mimar Sinan Town. Yıldırım/BURSA, Turkey
}

\section{ABSTRACT}

Background: The purpose of the present study was to compare the effects of two different clamping strategies for the construction of the proximal aortocoronary anastomoses on myocardial protection and postoperative outcomes during coronary artery bypass grafting (CABG) operations.

Methods: In this retrospective study, we examined prospectively collected data of patients who underwent CABG for a 3-year period. Two hundred consecutive patients, who were diagnosed with triple vessel coronary artery disease (CAD), were selected and divided into two groups. In Group 1 (single clamp) $(\mathrm{N}=100)$, venoaortic proximal anastomoses were performed using a single aortic cross-clamp, while in Group 2 (double clamp) $(\mathrm{N}=100)$, proximal anastomoses were performed by using an aortic side clamp. Operative and postoperative outcomes of the patients were compared between the two groups. The serum levels of myocardial damage biomarkers, creatine phosphokinase-MB (CPK-MB), and high sensitive Troponin (hsTnI) results were measured preoperatively, intraoperatively, and postoperatively $(6,12$, 24, and 48 hours).

Results: Patient demographics and characteristics were similar between the two groups. In Group 1, cross-clamp duration time (65 min versus $49 \mathrm{~min} ; P=.0001$ ) was longer. However, perfusion time (91 min versus $85 \mathrm{~min} ; P=.61$ ) was similar between the two groups. In Group 2, postoperative CK-MB levels were significantly higher intraoperatively $(P=.18), 6$ hours $(P=.22), 24$ hours $(P=.001)$, and 48 hours $(P=.001)$ than in Group 1. HsTnI was only significantly higher in Group 2 versus Group 1 at 24 hours $(P=.001)$ and 48 hours $(P=.01)$ postoperatively. Time of intensive care unit stay, duration of extubation, and length of hospital stay were similar in both groups.

Conclusion: The technique used for proximal anastomosis has a significant effect on perioperative results, especially on myocardial protection.

Received November 19, 2020; accepted December 10, 2020.

Correspondence: Cüneyt Eriş, MD, Assoc Prof, University of Health Sciences, Bursa Yuksek Ibtisas Training and Research Hospital, Department of Cardiovascular Surgery, Mimar Sinan Town, Emniyet Street, Yuldirım/ BURSA, Turkey; +90224-295-50-00; fax: +90224-275-67-67 (e-mail: dr_ceris@hotmail.com).

\section{INTRODUCTION}

Although coronary artery bypass grafting $(\mathrm{CABG})$ surgery with cardiopulmonary bypass (CPB) has been performed safely and effectively for a long time, multiple factors can lead to procedural myocardial injury during this procedure. Myocardial ischemia caused by inadequate myocardial preservation, the direct traumatic injury to the myocardium or any potential ischaemic injury during cardiac surgery causes cardiac functional and structural changes and directly affects the outcomes of the operation. For that reason, increases in cardiac biomarker values should be expected after all CABG procedures [Pegg 2011; Jørgensen 2014]. In some current studies, operational myocardial damage was observed among $32-44 \%$ of CABG patients [Ucak 2019; Rahimi 2009].

Myocardial protection is provided by the cardioplegic solutions given after the cross-clamping of the aorta during cardiac surgery. Some amount of myocardial ischemia develops in cases where adequate myocardial protection cannot be provided and as a result protein release from the injured cardiac myocytes occurs [Elassal 2020; Angelini 2002]. The preferred way to protect the myocardium is to administer adequate cardioplegic solution in the appropriate way to the whole myocardium but this route is limited to severe coronary artery stenosis [Sanjay 2003].

Another important indicator of achieving favorable results of CABG is the surgical technique [Hausenloy 2012]. Two different clamping strategies have been used for the construction of the proximal aorto-coronary anastomoses: single clamping or double clamping. In the single clamp technique, a veno-aortic proximal anastomosis is performed after each of the distal anastomosis. Thus, the cardioplegic solution given will nourish the myocardial tissue from both the native coronary artery and the newly constructed venous graft. In the double-clamp technique, after all distal anastomoses are completed, the aortic crossclamp is released and the proximal veno-aortic anastomoses are made with the clamp placed partially on the aorta. During this period, none of the venous grafts are filled with blood. Therefore, the heart is exposed to a relatively prolonged period of ischemia. After the aortic cross-clamp is removed, the heart starts to beat and when the internal mamarian artery graft is used, the heart is fed through this graft. Myocardial protection is provided only through obstructed or narrowed native arteries with cardioplegic 
Table 1. Patient demographics and preoperative data

\begin{tabular}{|c|c|c|c|}
\hline Variables & $\begin{array}{c}\text { Group } 1 \\
(N=100)\end{array}$ & $\begin{array}{c}\text { Group } 2 \\
(\mathrm{~N}=100)\end{array}$ & $P$ \\
\hline Age (years), mean $\pm S D$ & $61.1 \pm 8.76$ & $59.1 \pm 9.24$ & .13 \\
\hline Male gender, $\mathrm{n}(\%)$ & $77(77)$ & $82(82)$ & .63 \\
\hline Body mass index, mean $\pm S D$ & $28.2 \pm 5.4$ & $29.6 \pm 6.5$ & .61 \\
\hline Diabetes mellitus, $\mathrm{n}(\%)$ & $20(20)$ & $22(22)$ & .72 \\
\hline Hypertension, n (\%) & $37(37)$ & $35(35)$ & .72 \\
\hline History of smoking, n (\%) & $41(41)$ & $40(40)$ & .88 \\
\hline Hyperlipidemia, n (\%) & $40(40)$ & $36(36)$ & .56 \\
\hline Previous myocardial infarction, $\mathrm{n}(\%)$ & $38(38)$ & $42(42)$ & .68 \\
\hline Preoperative EF (\%), mean \pm SD & $50.5 \pm 9.54$ & $51.0 \pm 8.54$ & .66 \\
\hline EUROscore, mean $\pm S D$ & $1.9 \pm 1.50$ & $2.1 \pm 1.51$ & .42 \\
\hline
\end{tabular}

EF, ejection fraction; EUROscore, European System for Cardiac Operative Risk Evaluation; SD, standard deviation

solutions given between the distal anastomoses, causing an insufficient amount of solution to reach the distal of the stenotic coronary arteries.

Although the neurological outcomes of the use of aortic single or double clamps in on-pump CABG have extensively been investigated before, there are few studies about their effects on myocardial protection and functioning. In our study, we predominantly investigated the effects of clamping techniques on myocardial protection and ventricular functions during on-pump CABG. Furthermore, we compared the postoperative early outcomes of the two different aortic clamping techniques after on-pump CABG.

\section{MATERIALS AND METHODS}

In this study, patients who had undergone elective isolated CABG using CPB at Bursa Yuksek Ihtisas Education and Research Hospital, Bursa, Turkey, between January 2018 and June 2020, retrospectively were evaluated. During this time period, 330 patients underwent CABG, and 100 patients in each group who met the study criteria were included in this study, respectively. Patients were divided into two groups, according to the first operating surgeon. Patients in Group $1(\mathrm{~N}=100)$ were operated on by a surgeon, who prefers to use a single-clamp technique, whereas patients in Group $2(\mathrm{~N}=100)$ were operated on by another surgeon, who prefers double-clamp technique for the proximal anastomosis. All cases were operated by two surgeons (CE and $\mathrm{BE}$ ).

The study was planned, and approval of the Bursa Yuksek Ihtisas Education and Research Hospital Ethics Committee was obtained. Preoperative, operative, and postoperative variables and results were obtained from our
Table 2. Preoperative features of the patients

\begin{tabular}{lccc}
\hline Variables & $\begin{array}{c}\text { Group 1 } \\
(\mathrm{N}=100)\end{array}$ & $\begin{array}{c}\text { Group 2 } \\
(\mathrm{N}=100)\end{array}$ & $P$ \\
\hline Cross-clamp time (min), mean $\pm \mathrm{SD}$ & $65.6 \pm 24.62$ & $49.4 \pm 13.43$ & .001 \\
Perfusion time (min), mean $\pm \mathrm{SD}$ & $91.3 \pm 22.49$ & $85.5 \pm 20.88$ & .61 \\
Number of bypass grafts, $\mathrm{n}(\%)$ & - & - & .65 \\
2XCABG & $0(0)$ & $1(1)$ & \\
3XCABG & $52(52)$ & $53(53)$ & \\
4XCABG & $39(39)$ & $40(40)$ & \\
5XCABG & $9(9)$ & $6(6)$ & \\
Defibrillation, $\mathrm{n}(\%)$ & $39(39)$ & $30(30)$ & .18 \\
Inotropic agent needed, $\mathrm{n}(\%)$ & $30(30)$ & $29(29)$ & .87 \\
Intra-aortic balloon pump use, $\mathrm{n}(\%)$ & $3(3)$ & $4(4)$ & .70 \\
Re on pump, $\mathrm{n}(\%)$ & $2(2)$ & $4(4)$ & .40 \\
Extubation time (h), mean $\pm \mathrm{SD}$ & $11.2 \pm 3.67$ & $10.8 \pm 3.40$ & .42 \\
Intensive care stay (day), mean $\pm \mathrm{SD}$ & $2.3 \pm 0.79$ & $2.3 \pm 0.78$ & .92 \\
Discharge time (day), mean $\pm \mathrm{SD}$ & $7.4 \pm 1.28$ & $7.6 \pm 1.95$ & .41 \\
Postoperative EF (\%), mean $\pm \mathrm{SD}$ & $48.0 \pm 7.03$ & $48.04 \pm 7.86$ & .97 \\
Postoperative CVA, $\mathrm{n}(\%)$ & $2(2)$ & $3(3)$ & .65 \\
Postoperative atrial fibrilation, $\mathrm{n}(\%)$ & $10(10)$ & $15(15)$ & .28 \\
Postoperative MI, $\mathrm{n}(\%)$ & $1(1)$ & $2(2)$ & .40 \\
In hospital death, $\mathrm{n}(\%)$ & $1(1)$ & $2(2)$ & .40 \\
\hline & & & \\
\hline
\end{tabular}

CABG, coronary artery bypass graft; EF, ejection fraction; CVA, cerebrovascular accident; MI, myocardial infarction

hospital's computerized database. Data collection was based on hospital clinic records, anesthesia records, perfusion records, and intensive care data and observational records. All of the patients were suffering from triple vessel coronary artery disease with different occlusion degree of the left anterior descending (LAD) or other coronary arteries. Patients, who underwent CABG with at least three or more vascular grafts, were included in the study. Carotid arteries of all patients were routinely examined by carotid Doppler ultrasonography preoperatively. We excluded the patients with valvular heart diseases, atrial fibrilation, emergency cardiac operations, those who had undergone additional operation as well as CABG (such as ventricular aneurismectomy, coronary endarterectomy, carotid endarterectomy, mitral valve replacement, or aortic valve replacement), those who had a history of stroke, transient ischaemic attack or myocardial infarction within the last two weeks before the operation, those who had clinical evidence of carotid and peripheral vascular disease. We also excluded reoperations, patients who had preoperatively poor left ventricular function (ejection fraction $<30 \%$ ), renal failure (creatinine $>1.5 \mathrm{mg} / \mathrm{dL}$ ), or abnormal liver function (liver enzyme levels of greater than two times the upper limit of normal), and patients who were more than 75 years old. 
Table 3. Creatine Kinase MB values of the patients

\begin{tabular}{lccc}
\hline Time & Group 1 $(\mathrm{N}=100)$ & Group 2 $(\mathrm{N}=100)$ & $P$ \\
\hline Preoperative & $1.5 \pm 0.63$ & $1.6 \pm 0.73$ & .46 \\
Intraoperative & $19.2 \pm 12.76$ & $14.9 \pm 12.88$ & .018 \\
6 hours & $37.6 \pm 19.82$ & $46.4 \pm 32.47$ & .022 \\
12 hours & $71.5 \pm 38.36$ & $75.9 \pm 51.34$ & .49 \\
24 hours & $76.1 \pm 57.97$ & $115.4 \pm 63.06$ & .001 \\
48 hours & $147.9 \pm 106.61$ & $244.7 \pm 146.31$ & .001
\end{tabular}

Demographic and preoperative features (age, sex, smoking, hypertension, diabetes mellitus, routine hypertension, smoking history, hypercholesterolaemia and preoperative left ventricular ejection fraction (LVEF), etc.), operative (cross-clamp times, perfusion times, number of bypass grafts used, left internal mamarian artery (LIMA) use, defibrilation needs) and postoperative data (intraaortic baloon pump (IABP) requirement, inotrope agent requirement, perioperative electrocardiogram changes, development of new atrial fibrillation, extubation times, intensive care unit (ICU) stay times and discharge times, etc.) were recorded. Blood samples were drawn from each patient one day before the operation, after protamine administration intraoperatively, 6 hours, 12 hours, 24 hours and 48 hours postoperatively to measure the myocardial biomarkers. HsTnI and CK-MB were measured by a Roche Cobas ${ }^{\circledR} 8000$ analyser (Roche Diagnostics, Mannheim, Germany). Left ventricular ejection fractions, which was an indicator of cardiac functions, were compared preoperatively and postoperatively before discharge with 2-dimensional echocardiography.

Surgical technique: Cold blood cardioplegia was given antegradely after each distal and proximal anastomosis in Group 1 and cold saline was used for topical cooling in both groups. LIMA-LAD anastomosis was performed for $98 \%$ of the patients in each group. Veneous grafts were used in all other bypasses. In both groups, warming was initiated at the beginning of the LIMA-LAD coronary artery anastomosis. Before releasing the aortic cross-clamp, a terminal dose of warm blood cardioplegia was given in every procedure. In Group 1, the proximal anastomoses were performed after each distal anastamose, while the aorta was cross clamped. In Group 2, all proximal venoaortic anastomoses were constructed by placing a single aortic side occluding clamp, meanwhile the heart was beating and LIMA, if used, began to work.

Statistical analysis: Statistical analyses were performed with the Statistical Package for the Social Sciences version 23.0 (SPSS Inc, Chicago, Illinois, USA). Continuous variables are presented as means \pm standard deviations, whereas categorical variables are presented as percentages. Inter-group comparisons were made by Student's t-test (for normally distributed data) or the Mann-Whitney U test (for other data). Categorical varieties were compared by the chi-square or Fisher's exact test. A $P$ value less than .05 was accepted as statistically significant for all comparisons.
Table 4. High-Sensitivity Troponin values of the patients

\begin{tabular}{lccc}
\hline Time & Group 1 $(\mathrm{N}=100)$ & Group 2 $(\mathrm{N}=100)$ & $P$ \\
\hline Preoperative & $13.2 \pm 7.32$ & $14.9 \pm 8.84$ & .130 \\
Intraoperative & $184.7 \pm 106.52$ & $170.1 \pm 108.62$ & .336 \\
6 hours & $445.1 \pm 264.71$ & $475.1 \pm 376.60$ & .516 \\
12 hours & $535.2 \pm 269.91$ & $535.9 \pm 359.69$ & .98 \\
24 hours & $299.7 \pm 175.50$ & $436.5 \pm 235.13$ & .001 \\
48 hours & $149.6 \pm 105.94$ & $248.8 \pm 147.98$ & .001
\end{tabular}

\section{RESULTS}

Data from the 100 consecutive patients in whom proximal anastomosis had been performed with single cross clamping were evaluated as Group 1 ( $N=100 ; 77$ males, 23 females; age $61.10 \pm 8.76$ years; range of $40-75$ years). The data from the other 100 consecutive patients in whom proximal anastomosis had been performed with double clamping were evaluated as Group $2(\mathrm{~N}=100 ; 82$ males, 18 females; age $59.17 \pm 9.24$ years; range of $40-75$ years). The data for these two groups were compared. Patient demographics and characteristics were similar between the two groups (Table 1).

Operative and postoperative clinical data are summarized in Table 2. The aortic cross-clamping times were significantly longer in Group 1 (single clamp) than in Group 2 (double clamp) (65.6 $\pm 24.62 \mathrm{~min}$ versus $49.4 \pm 13.43 \mathrm{~min} ; P=.001)$ as expected but there were no significant differences in the total perfusion time $(91.3 \pm 22.49 \mathrm{~min}$ versus $85.5 \pm 20.88 \mathrm{~min} ; P$ $=.61$ ) between the groups. At the time of cross-clamp release more cases in Group 1 (39\%) needed defibrillation to return to normal sinus rhythm compared with Group 2 (30\%) but there was no significant difference $(P=.18)$. Inotropic agents support were needed 30\% in Group 1 and 29\% in Group 2 $(P=.87)$ at the time of weaning from CPB. Two cases $(2 \%)$ in Group 1 and four cases (4\%) in Group 2 needed re-initiation of $\mathrm{CPB}$, due to hemodynamic instability. Postoperative ejection fractions were not significantly different between the two study groups $(48.0 \pm 7.03$ versus $48.04 \pm 7.86 ; P=.97)$. We also found that postoperative cerebrovascular accident (CVA) rates $(2 \%$ versus $3 \% ; P=.65)$, postoperative IABP $(3 \%$ versus $4 \% ; P=.70)$ and inotropic agent need $(30 \%$ versus $29 \% ; P=$ .87) was not significantly different. In the single-clamp group, the need for postoperative defibrillation after the clamp was removed was higher, but this difference did not reach a statistical significance $(39 \%$ versus $30 \% ; P=.18)$.

There was no statistical difference between the two groups in preoperative hsTnI and CK-MB levels. In Group 2, postoperative CK-MB levels were statistically significantly higher after the protamin administration intraoperatively $(P=.18), 6$ hours $(P=.22), 24$ hours $(P=.001)$, and 48 hours $(P=.001)$ postoperatively than in Group 1 (Table 3), whereas hsTnI was only statistically significantly higher in Group 2 versus Group 1 at 24 hours $(P=.001)$ and 48 hours postoperatively $(P=.01)$ (Tables 3 and 4). 
One death occurred in Group 1 at the postoperative 12th day due to sepsis leading to multiorgan failure. Two deaths occurred in Group 2. The first of these patients had postoperative right hemiplegia and subsequently his neurological problem worsened, and he died on the 15 th postoperative day. The second patient died on the 17 th day because of multiorgan failure due to mediastenitis originated sepsis.

\section{DISCUSSION}

One of the most important issues for successful CABG is providing adequate protection of the myocardial tissue with the cardioplegic solutions through the severly stenosed coronary arteries during the aortic cross-clamping period. The extent of maldistribution of cardioplegia is responsible for the degree of myocardial damage that may occur during $\mathrm{CABG}$ procedures [Ali 2020]. In the literature, the incidence of perioperative myocardial damage after CABG has been reported between $5 \%$ and $20 \%$ and this varies according to the use of different definitions and diagnostic criteria [Chowdhury 2008].

We can group the causes of perioperative myocardial damage after CABG under two headings as graft-related and non-graftrelated ones. The graft-related factors are graft occlusion due to graft kinking or overstretching, failed anastomosis, or graft spasm. Non-graft-related causes include direct myocardial trauma caused by slings during manipulation of the heart, cold damage caused by ice slush application, inadequate cardioplegic perfusion and myocardial protection, incomplete revascularization, and distal coronary microembolization caused by air or debris [Scarsisi 2016; Crescenzi 2004].

Buckberg [Buckberg 1979] in 1979, recommended constructing the distal and proximal anastomosis sequentially under single aortic clamping period for achieving homogeneous cardioplegia delivery and cooling of all myocardial regions for adequate myocardial protection. Cold blood cardioplegia infusion from both native coronary arteries and newly constructed grafts allows a reduction in metabolic demand and continuous washing of lactic acid during ischemia. When the cross-clamp is removed, there is a maximal reactive hyperemia and a sudden maximal coronary artery reperfusion, resulting in complete washout of lactic acid accumulation and rapid regeneration of the substrates necessary for aerobic metabolism [Zakkar 2015].

On the other hand, Khoury et al. [Khouri 1988] found a significant decrease in myocardial $\mathrm{pH}$ and an increase in myocardial temperature because of inadequate coronary hyperemia and reperfusion when a side clamp was used to construct proximal anastomoses. They reported that this situation causes continued ischemia, increased lactic acid production and accumulation, and myocardial reperfusion injury resulting from the formation of free oxygen radicals.

During $\mathrm{CPB}$, cardioplegic solution administered antegradely from the aortic root is the preferred method to provide sufficient myocardial protection, but it may not be possible to protect the whole myocardium equally, especially in patients with severe coronary occlusions. Prolonged myocardial ischemia below approximately 20 minutes causes structural and biochemical damage of the myocardial cells and it causes a temporary decrease in myocardial contractility [Sirvinskas 2015]. If the restoration of blood flow is achieved over 45 minutes after myocardial ischemia, myocardial ischemic reperfusion injury may develop, and the resulting myocardial damage is much greater than the damage caused by ischemia alone [Turer 2010].

The incidence of perioperative myocardial infarction after CABG ranges from $2 \%$ to $15 \%$. The most important factor in this wide range can be explained by the absence of a specific gold standard test in the case of post-CABG infarction. In addition, electrocardiographic changes and elevation of cardiac enzymes can be seen postoperatively, even in patients without myocardial ischemia [Sobral 2006; Adam 2004]. It should be recognized that ST-segment deviation and $\mathrm{T}$ wave changes are common after CABG due to epicardial injury, and are not reliable indicators of myocardial ischaemia. However, ST-segment elevation with reciprocal ST-segment depression may be a more reliable finding of a potential ischaemic event [Thielmann 2017]. In our study, perioperative myocardial infarction occurred in one patient in Group 1 and two patients in Group 2 $(P=.40)$. Since the conditions of these patients were stable with medical treatment, no extra intervention was performed.

Adequate distribution of cardioplegic solution is extremely important, especially in patients with coronary artery disease. When partial aortic clamp is applied for proximal anastomoses after the cross-clamp is released from the aorta, reperfusion will be provided from stenotic native vessels and from the IMA anastomosis if it was performed, adequate reperfusion will not be achieved due to hypotension and IMA spazm in the early period [Daniel 3rd 2014]. In addition, in the single-clamp technique, unlike the double-clamp technique, cold blood cardioplegia is given additionally after each new proximal veno-aortic anastomosis and each anastomosis is activated in the early period, so adequate myocardial protection by achieving homogeneous cardioplegia delivery and cooling of all myocardial regions was established.

Many cardiac surgeons construct the proximal veno-aortic anastomoses during a single period of aortic side clamping, as in our Group 2 patients. Although some researchers hypothesized that constructing the proximal veno-aortic anastomoses one by one with multiple side biting techniques (sequential partial occluding aortic side biting technique) shortens ischemic time, they consequently provide better myocardial protection in patients undergoing CABG. In the study by Jelodar et al. [Jelodar 2017], they compared sequential partial occluding aortic side biting technique with single clamp technique and their results showed that in the study group 24-hours after the operation, serum levels of CPK-MB and troponin I were significantly lower compared with the single-clamp group. In our study, troponin and CK-MB values were found to be significantly lower in the single-clamp group compared with the side-clamp group in the postoperative period, and we concluded that the use of single-clamp method in on-pump CABG operations protects the myocardium better. Also, although there was a slight decrease in left ventricular ejection fractions in the postoperative period compared with the preoperative period, there was no significant difference. 
Postoperative stroke is a rare but major cause of morbidity and mortality in patients undergoing CABG. In on-pump CABG, the incidence of perioperative cerebrovascular accident (CVA) ranges from $1.5 \%$ to $5.2 \%$ in prospective studies and $0.8 \%$ to $3.2 \%$ in retrospective studies [D'Ascenzo 2012]. An important cause of postoperative cerebral atheroembolism is manipulation of the ascending aorta [Blauth 1995].

In the study of Mills et al, they reported that the rate of embolic events during clamp insertion and removal was reported respectively as $11 \%$ and $17 \%$ in the side-clamp group. This ratio was reported as $0.1 \%$ and $9 \%$ in the singleclamp group, respectively [Mills 1993]. On the other hand, it is not correct to attribute all postoperative cerebral complications to aortic clamping alone. Aortic cannulation/decanulation, aortic punching, proximal anastomosis construction and insufficient dearing strategies are involved in the etiology of neurological complications after CABG. It can also be seen in patients with atrial fibrillation or intracranial vascular stenosis. In our study, two patients in the single-clamp group and three patients in the side-clamp group developed SVA, and no significant difference $(P=.65)$ was found between the groups. We think that the reason for the lack of significant difference between the two groups is that, our patient population was relatively young. In addition, cases with peripheral vascular disease and complicated cases with additional surgical procedures were excluded from the study. In order to minimize the risk of thromboembolism and to avoid unexpected events, we examined the ascending aorta with digital palpation, which can easily detect an obvious abnormality, such as porcelain aorta or aortic calcification before the cannulation and before aortic punching. If we were in doubt about any aortic calcification or plaque, we avoid using side clamps and performed proximal anastomoses with using only single-clamp technique.

Arrhythmias, especially atrial fibrillation, are quite common after coronary artery bypass surgery, and their prevalence varies between 4-34\% [Erdolu 2020]. In our study, after the removel of the cross clamp, the need for defibrillation was slightly higher in the single-clamp group (39\% versus $30 \%$; $P=.18)$, but it was not statistically significant. We think the reason fibrillation after cross-clamping was more common in the single-clamp group can be attributed to the fact that the amount of potassium-rich cardioplegic solutions given after every proximal and distal anastomosis was much higher than the other group, which was given cardioplegia only after distal anastomoses. Postoperative atrial fibrillation was seen in $10 \%$ in the single-clamp group and $15 \%$ in the side-clamp group, and there was no statistically significant difference ( $P$ $=.28$ ) between the groups.

Another risk of using side clamp during the suturing of proximal veno aortic graft anastomoses is that it may cause aortic vascular injury and even aortic dissection [Ram 2020]. This risk increases even more with sequential clamps. In our study, partial aortic dissection occurred in one patient in the side-clamp group and a dacron graft was implanted in the ascendan aorta.

Although advocates of the two-clamp technique argue that no preservation technique is better than early removal of the cross-clamp and releasing of the internal mammary artery graft as soon as possible provides a positive contribution to feeding of the myocardium, in our study the cross-clamp time was found to be significantly longer but no significant difference was detected at the total time of perfusion in the singleclamp group. The conclusion we draw here is not the crossclamp time but how well the myocardium is protected during the aorta is cross-clamped.

The only drawback of this study is that it was retrospective, and nonrandomized. In order to more clearly evaluate the effect of the clamping strategy on myocardial protection; patients in the mild to moderate risk group and those with no additional pathologies were included in the study. The effects of aortic clamping, especially in the high-risk group or in the patient group undergoing combined surgery, are worth investigating.

\section{CONCLUSION}

In summary, the proximal veno-aortic reconstruction technique significantly affects the perioperative outcomes after CABG. The single cross-clamp method is a simple and safe technique that prepares the myocardium for reperfusion due to a possible more uniform cardioplegia delivery and distribution, provides better myocardial protection and also provides better cerebral protection due to less aortic manipulation and atheroembolic events. We believe that the advantage of single-clamp method will be more pronounced especially in the high-risk group of patients with peripheral vascular disease, with poor left ventricular function and the need for additional cardiac interventions due to valvular problems besides CABG.

\section{REFERENCES}

Adam DH, Filsoufi F, Antmann EM. 2004. Medical management of the patient undergoing cardiac surgery. In: Zipes DP, Libby P, Bonow R, Braunwald E, eds. Braunwald's heart disease: a textbook of cardiovascular medicine. 7th ed. Philadelphia: WB Saunders; p.1993-2019.

Ali M, Moeen M, Paras I, et al. 2020. Cardio-Protective Effects of Multiport Antegrade Cold Blood Cardioplegia Versus Antegrade Cold Blood Cardioplegia in Patients With Left Ventricular Systolic Dysfunction Undergoing Conventional Coronary Artery Bypass Grafting. Cureus. 12(9): e10308.

Angelini GD, Taylor FC, Reeves BC, et al. 2002. Early and midterm outcome after off-pump and on-pump surgery in beating heart against cardioplegic arrest studies (BHACAS 1 and 2): a pooled analysis of two randomized controlled trials. Lancet. 359:1194-9.

Blauth CI. Macroemboli and microemboli during cardiopulmonary bypass. 1995. Ann Thorac Surg. 59:1300-3.

Buckberg CD. 1979. A proposed solution to the cardioplegic controversy. J Thorac Cardiovasc Surg. 7.

Chowdhury UK, Malik V, Yadav R, et al. 2008. Myocardial injury in coronary artery bypass grafting: On-pump versus off-pump comparison by measuring high-sensitivity C-reactive protein, cardiac troponin I, hearttype fatty acid-binding protein, creatine kinase- $\mathrm{MB}$, and myoglobin release. J Thorac Cardiovasc Surg. 135:1110-9. 
Crescenzi G, Cedrati V, Landoni G, et al. 2004. Cardiac biomarker release after CABG with different surgical technique. J Cardiothorac Vasc Anesth. 18:34-7.

D’Ascenzo F, Cavallero E, Moretti C, et al. 2012. Remote ischaemic preconditioning in coronary artery bypass surgery: A Meta-analysis. Heart. 98(17):1267-71.

Daniel 3rd WT, Kilgo P, Puskas JD, et al. 2014. Trends in aortic clamp use during coronary artery bypass surgery: effect of aortic clamping strategies on neurologic outcomes." The Journal of thoracic and cardiovascular surgery. J Thorac Cardiovasc Surg. 147(2):652-7.

Elassal AA, Al-Ebrahim K, Al-Radi O, et al. 2020. Myocardial Protection by Blood-Based Del Nido versus St. Thomas Cardioplegia in Cardiac Surgery for Adults and Children. In The Heart Surg Forum. 23(5):E689-E695.

Erdolu B, As AK, Engin M. 2020. The Relationship between the HATCH Score, Neutrophil to Lymphocyte Ratio and Postoperative Atrial Fibrillation After Off-Pump Coronary Artery Bypass Graft Surgery. Heart Surg Forum 23(1);E088-E092.

Hausenloy DJ, Boston-Griffiths E, Yellon DM. 2012. Cardioprotection during cardiac surgery. Cardiovasc Res. 94(2):253-65.

Jelodar G, Zarrabi K, Nazifi S, et al. 2017. Comparison of Serum Myocardial Biomarkers Following Two Different Methods of Venoaortic Connection in Coronary Artery Bypass Grafting: A Randomized Clinical Trial. Zahedan J Res Med Sci. 19(7):e10740.

Jørgensen PH, Nybo M, Jensen MK, et al. 2014. Optimal cut-off value for cardiac troponin I in ruling out type 5 myocardial infarction. Interact Cardiovasc Thorac Surg. 18:544-550.

Khouri SF, Warner KG, Butler M, et al. 1988. The superiority of continuous cold blood cardioplegia in the metabolic protection of the hypertrophied human heart. J Thorac Cardiovasc Surg. 95(3):442-54.

Mills SA. Cerebral injury and cardiac operations. 1993. Ann Thorac Surg. 56:S86-91.

Pegg TJ, Maunsell Z, Karamitsos TD, et al. 2011. Utility of cardiac biomarkers for the diagnosis of type $\mathrm{V}$ myocardial infarction after coronary artery bypass grafting: Insights from serial cardiac MRI. Heart. 97:810-816.
Rahimi K, Banning AP, Cheng AS, et al. 2009. Prognostic value of coronary revascularisation-related myocardial injury: A cardiac magnetic resonance imaging study. Heart. 95:1937-1943.

Ram H, Dwarakanath S, Green AE, et al. 2020. Iatrogenic aortic dissection associated with cardiac surgery: A narrative review. Journal of Cardiothoracic and Vascular Anesthesia. Online ahead of print.

Sanjay OP, Srikrishna SV, Prashanth P, et al. 2003. Antegrade versus antegrade with retrograde delivery of cardioplegic solution inmyocardial revascularisation. A clinical study in patients with triple vessel coronary artery disease. Ann Card Anaesth. 6(2):143-8.

Scarsini R, Zivelonghi C, Pesarini G, et al. 2016. Repeat revascularization: percutaneous coronary intervention after coronary artery bypass graft surgery. Cardiovascular Revascularization Medicine. 17(4):272-278.

Sirvinskas E, Kinderyte A, Trumbeckaite S, et al. 2015. Effects of sevoflurane vs. propofol on mitochondrial functional activity after ischemiareperfusion injury and the influence on clinical parameters in patients undergoing CABG surgery with cardiopulmonary bypass. Perfusion. 30:590-595.

Sobral MLP, Santos GG, Santos LAS, et al. 2006. Comparative randomized study of the immediate outcomes of patients with radial arteries proximally anastomosed to the aorta or as a composite graft. Rev Bras Cir Cardiovasc. 21(1):35-41.

Thielmann M, Sharma V, Al-Attar N, et al. 2017. ESC Joint Working Groups on Cardiovascular Surgery and the Cellular Biology of the Heart Position Paper: Peri-operative myocardial injury and infarction in patients undergoing coronary artery bypass graft surgery. Eur Heart J. 38:2392-2411.

Turer AT, Hill JA. 2010. Pathogenesis of myocardial ischemia-reperfusion injury and rationale for therapy. Am J Cardiol. 106(3):360-8.

Ucak HA, Uncu H. 2019. Impact of Coronary Collateral Circulation of Perioperative Myocardial Damage in High-Risk Patients Undergoing Coronary Artery Bypass Grafting Surgery. Heart Surg Forum. 11;22(5):E375-E379.

Zakkar M, Guida G, Suleiman MS, et al. 2015. Cardiopulmonary bypass and oxidative stress. Oxid Med Cell Longev. 2015:189863. 